\section{Scalable System for Increasing Patient-Care Capacity in Large-Scale Disasters: The Modular Emergency Medical System}

\section{Doyle; J. Atas; A. Mahar}

Region 2S Medical Biodefense Network, USA

When large-scale disasters strike, hospitals and emergency centers can be overwhelmed by patient loads. A system to use off-hospital medical screening, minor care, prophylaxsis and mid-level general ward care in a scaleable fashion can help to remove the burden of the lower levels of medical care from some of the hospitals. Draft plans to use a modular system to augment medical care are being developed. The modular emergency medical system (MEMS) plans to direct regions to examine and inventory regional resources and to plan for operation of off-hospital site facilities. These areas would be staffed and equipped to cover larger areas than local emergency preparedness jurisdictions that could not be handled with mutual aid, and movement of patients into other unaffected jurisdictions. These plans originally were planned for bioterrorism casualties, but would generalize to other large-scale incidents. Plans are being drafted for Neighborhood Emergency Help Centers (NEHC) that would serve as triage points for less ill patients, vaccination and/or prophylaxsis centers, and points for screening, education, and follow-up of home-based care and quarantined persons. Staffing would draw on credentialed volunteers from non-acute care sites and public heath, as well as retirees and part-time workers.

A cooperative agreement with a coalition of public health and hospital partners in planning and drafting plans and processes is essential.

Patients who do not require extensive nursing or respiratory care would be triaged to an Acute Care Center (ACC) These Centers are envisioned as providing midlevel care to patients that are able to be discharged from a hospital to ward level care and for patients needing ward care including medications and oxygen. Cardiac monitoring is not planned in this setting. This setting would allow hospitals to focus on more seriously ill or injured and patients who require special isolation or ventilatory support. The ACCs could be used to cohort patients with a specific infectious problem. Credentialed volunteers who have additional in-service training in the operation of Acute Care Centers would provide the medical care. A coalition of hospitals and regional emergency planners as well as emergency preparedness planners and governmental officials would be needed to plan, execute, and coordinate these Centers.

Supply and re-supply as well as equipment and facility locations and utilization require advance planning. Memoranda of understanding between facilities and networks contributing to constructing and maintaining the Centers must be in place in advance of operation. Drills should be conducted in order to test the transportation between facilities and communication and equipment. Networks of providers and government emergency preparedness planners and all entities that will contribute should be formed to assist integration with local planning.
Keywords: care; disasters; emergency medical system; large-scale; patient; scalable; system

Pretosp Disast Med 2005;20(3):s137

The Development of a National Model of Care for Burn Patients to Support the Activation of the Australian Burn Disaster Plan (Ausburn Plan)

F. Wood, ${ }^{1}$ D. Cooper; ${ }^{2}$ D. Edgar, ${ }^{3}$ A. Robertson ${ }^{3}$

1. Royal Perth Hospital, Perth, Australia

2. Ambulance Service of New South Wales, Australia

3. Department of Health, Western Australia, Australia

The emergency and disaster management arrangements in Australia are based upon the comprehensive approach involving prevention, preparedness, response, and recovery. Standards, education, and training of appropriate personnel are integral components of all the agencies' coordinated response.

Education and training support the disaster response and need to be inclusive of not only core burn care, but include disaster management strategies as well. The Ausburn Plan is based on amplification of core capabilities.

Burn care personnel must be trained in disaster response and be informed of plans at local and national levels.

Strategies developed and proposed to support the disaster plan activation will be discussed.

Keywords: Ausburn Plan; burn patients; disaster plan; emergency; management; strategies

Prebosp Disast Med 2005;20(3):s137

\section{Disaster Preparedness in Australian Emergency} Departments: Has It Improved and How Real Is It? P.J. Aitken,${ }^{1}$ D. Caldicott, ${ }^{2}$ N. Edwards, ${ }^{2}$ A. Eliseo, ${ }^{2}$ C. $L e e^{2}$

1. James Cook University School of Public Health, Australia 2. Royal Adelaide Hospital, Australia

Introduction: This paper reviews the disaster preparedness of emergency departments (EDs) in Australia with a focus on education, training, and resource management for both "conventional" and chemical, biological, and radiological (CBR) disasters. The approximate amount of change during the last two years also was determined.

Methods: In January 2004, a survey was mailed to directors of all emergency departments in Australia who were accredited for training by the Australasian College for Emergency Medicine (ACEM). The survey results were compared to standards and previous studies to determine changes in preparedness.

Results: The survey achieved an $86 \%$ response rate (consistent across demographic variables) and can be seen as representative of Australian emergency departments. Planning, education, exercises, and training all were more likely to occur for conventional than CBR disasters. Confidence in performance ability was less for CBR disasters, with most emergency departments (72\%) feeling they only could manage 10-20 patients during the first two hours. The maximum level personal protective equipment (PPE) available was level $\mathrm{C}$ or better in $66 \%$ of the emergency departments. While some type of decontamination facility was present in most, 
the type varied considerably. A number of emergency departments were prepared to send staff outside to manage patients $(43 \%)$ or into the hot zone (11\%). This did not correlate with the level of PPE or decontamination facility available.

Conclusion: Disaster preparedness has improved in Australian EDs over the past two years in some areas, most notably equipment availability for PPE and decontamination. However, there are concerns about the perceived or actual levels of preparedness of some emergency departments and the need for funded education and training.

Keywords: Australia; chemical, biological, and radiological (CBR); decontamination; disasters; education; emergency departments; personal protective equipment; preparedness; training

Prehosp Disast Med 2005;20(3):s137-s138

\section{Role of Hospitals in Disasters}

M.W. Chaffee; ${ }^{1}$ N.S. Oster ${ }^{2}$

1. United States Navy, USA

2. The Methodist Hospital, Brooklyn, New York USA

Hospitals are depended upon to be a cornerstone of response to disaster, but many hospitals are not adequately prepared to respond effectively. This presentation will address the impact of disaster on hospitals, the evolving role of the health system in national security, and public expectations.

A discussion of current practices in hospital preparedness will examine why hospitals are vulnerable to disaster. Forces impacting hospital preparedness will be identified. The critical elements of a solid hospital preparedness program will be described along with chronic pitfalls in hospital preparedness.

The current state of the science in hospital preparedness for acute-onset disasters will be examined and gaps in current knowledge will be addressed.

The level of preparedness in dozens of hospitals in the United States and in other nations has been analyzed. A hospital preparedness program that was an award finalist in the 2004 Harvard University/Mitretek Innovations in American Government Homeland Security award was led by the authors.

Keywords: assessment; current practices; disasters; hospitals; preparedness

Prehosp Disast Med 2005;20(3):s138

\section{Resistance Factors to Rapid Response in Natural Disaster Scenarios \\ C.R. Jagger \\ Binscombe Medical Centre, United Kingdom}

Introduction: The frequency of natural disasters is increasing. Rapid response on a local, national, and sometimes, international scale is required to offset increasing levels of mortality and material loss. Many factors can frustrate or impede the rapid response of individuals and relief organizations. Each organization will encounter difficulties regarding rapid movement of personnel and appropriate equipment to a natural disaster zone. These may be specific to a particular organization, but common obstacles to rapid response exist as well. The hypothesis is that there will be common obstacles to rapid response encountered by the various relief organizations. This study seeks to gauge the opinions of those engaged in response strategies to natural disasters, regarding these resistance factors. This study is qualitative in approach and attempts to establish a priority "hit list" which could serve as material for intervention and improvement for the disaster relief organizations.

Methods: A research tool (questionnaire) was constructed based on findings from extensive meetings and discussions with those engaged in disaster relief, together with observations from first-hand experiences in the emergency relief effort in El Salvador after the earthquakes of 2001. The questionnaire was circulated to members of the World Association for Disaster and Emergency Medicine (WADEM), other emergency relief agencies, and nongovernment organizations, after an appropriate pilot study using a random sample of WADEM members.

The circulation of the questionnaires and collation of material from the responses is ongoing, but the bulk of the responses were received during 2002-2003.

Results: Responses were received from four main sources-WADEM, Tear Fund (NGO), some United Nations departments, and Search and Rescue teams. The WADEM members from 17 countries completed the questionnaires. Fourteen of the 46 "resistance factors" to rapid response listed on the questionnaire were highlighted by each respondent consistently.

Conclusions: The attention to resistance factors to rapid response using the sampling tool yielded a positive response. A pattern of consensus has emerged which is worthy of further study. The magnitude of a given event versus the state of readiness and capacity of the local hospital services and defense organizations is a key issue. Disaster mitigation and preparedness is now high on the agenda regarding disaster response. It behoves all engaged in disaster response to examine the efficiency of their particular organization and the systems employed, and to ask the question: "Could improvements be made in the response time to a given event and could resistance factors to rapid response be overcome?"

Keywords: natural disasters; relief; response

Prebosp Disast Med 2005;20(3):s138

Emergency Air Medical Services in Taiwan: Long Distance Aeromedical Evacuation of Patients From a Remote Island by a Chartered, Fixed-Wing Air Ambulance
S.H. Tsai, ${ }^{1}$ H.R. Wu, ${ }^{2}$ L.J. Cbi, ${ }^{3}$ L.H. Lu; ${ }^{4}$ T.S. Chen; ${ }^{5}$
M.R. Lin; ${ }^{1}$ W.T. Chiu ${ }^{6}$
1. Taipei Medical University, Taiwan
2. Chung Shan Hospital, Taiwan
3. International SOS, Taiwan
4. Department of Emergency Medicine, Mackay Memorial, Taiwan
5. Bureau of Public Health and Environmental Protection, Taiwan
6. Institute of Injury Prevention and Control, Taiwan

Background: There is an increasing demand for emergency air medical services (EAMS) in Taiwan. Geographic limitations, as well as physician shortages, on some outer islands of Taiwan have resulted in increased EAMS development on remote islands. 